\title{
Pulsed neutron time-dependent intensity modulation for quasi- elastic neutron scattering spectroscopy
}

\section{$\operatorname{AUTHOR}(\mathrm{S})$ :}

Oda, T.; Hino, M.; Kitaguchi, M.; Geltenbort, P.; Kawabata, Y.

\section{CITATION:}

Oda, T. ... [et al]. Pulsed neutron time-dependent intensity modulation for quasi-elastic neutron scattering spectroscopy. Review of Scientific Instruments 2016, 87(10): 105124.

\section{ISSUE DATE:}

2016-10-27

\section{URL:}

http://hdl.handle.net/2433/259243

\section{RIGHT:}

This article may be downloaded for personal use only. Any other use requires prior permission of the author and AIP Publishing. This article appeared in ['Review of Scientific Instruments' 87, 105124 (2016)] and may be found at

https://aip.scitation.org/doi/10.1063/1.4965835; The full-text file will be made open to the public on 27 October 2017 in accordance with publisher's 'Terms and Conditions for SelfArchiving' 


\title{
Pulsed neutron time-dependent intensity modulation for quasi-elastic neutron scattering spectroscopy
}

\author{
T. Oda, ${ }^{1, a)}$ M. Hino, ${ }^{2}$ M. Kitaguchi, ${ }^{3,4}$ P. Geltenbort, ${ }^{5}$ and Y. Kawabata ${ }^{2}$ \\ ${ }^{1}$ Department of Nuclear Engineering, Kyoto University, Kyoto 615-8540, Japan \\ ${ }^{2}$ Research Reactor Institute, Kyoto University, Kumatori 590-0494, Japan \\ ${ }^{3}$ Department of Physics, Nagoya University, Nagoya 464-8602, Japan \\ ${ }^{4}$ Center for Experimental Studies, KMI, Nagoya University, Nagoya 464-8602, Japan \\ ${ }^{5}$ Institut Laue-Langevin, 38042 Grenoble, France
}

(Received 19 May 2016; accepted 8 October 2016; published online 27 October 2016)

\begin{abstract}
We propose a basic formula and demonstration for a high-resolution quasi-elastic neutron scattering (QENS) by combining the time-of-flight (TOF) method with Modulation of Intensity by Zero Effort (MIEZE) type neutron spin echo spectroscopy. The MIEZE technique has the potential to develop a unique approach to study on slow dynamics of condensed matter; however, the energy resolution is limited owing to the hypersensitivity of the MIEZE signal contrast to the echo condition, which is strongly affected by the alignment of the instruments and the sample. The narrow allowance of the optimal alignment is a major obstacle to the wide use of this technique. Combining the TOF method with MIEZE (TOF-MIEZE), the hypersensitivity of MIEZE signals is significantly alleviated with a short pulsed beam. This robustness is very useful to optimize experimental alignments and enables accurate measurements of QENS. The experimental results demonstrate the characteristic of the TOF-MIEZE technique and are well described by the formula presented in this study. Published by AIP Publishing. [http://dx.doi.org/10.1063/1.4965835]
\end{abstract}

\section{INTRODUCTION}

Neutron scattering is a very useful technique for studies of various material systems. The neutron spin echo (NSE) method proposed by Mezei is a powerful tool to investigate slow dynamics of condensed matter. ${ }^{1,2}$ It measures tiny velocity changes of neutrons scattered by a sample and directly obtains the intermediate scattering function $S(\boldsymbol{Q}, \tau)$ with high energy resolution. The neutron resonance spin echo (NRSE) method is a type of NSE spectroscopy using resonance spin flippers (RSFs). ${ }^{3,4}$ In the NRSE method, the Larmor precession coils of the Mezei-type NSE instruments are replaced by pairs of $\pi$-RSFs. These instruments have devices to cause precession before and after the sample. Unlike them, the Modulation of Intensity by Zero Effort (MIEZE) technique, ${ }^{5}$ a variant of NRSE, omits a pair of RSFs after the sample. In the technique, an essential spin analyze is done before the sample and there is no optical component after the sample. The spin eigen-state of neutrons incident on the sample is only one. This feature enables us to combine the MIEZE technique and polarimetry analysis, and makes the sample environment very flexible. ${ }^{6}$ It is also possible to synchronize a MIEZE measurement with the sample environment, for example, a pulse magnetic field or laser excitation, as the MIEZE signal is measured at each neutron pulse. The MIEZE technique has a great potential to discover new fields of the quasi-elastic neutron scattering spectroscopy with increasing neutron intensity. For these reasons, the construction of the MIEZE and NRSE spectrometers has been launched at BL06

\footnotetext{
a)Present address: Research Reactor Institute, Kyoto University, Kumatori 590-0494, Japan. Electronic mail: t_oda@ @ri.kyoto-u.ac.jp
}

at the Materials and Life Science Facility (MLF) of the Japan Proton Accelerate Research Complex (J-PARC). ${ }^{7,8}$ An MIEZE instrument is also proposed at the European Spallation Source (ESS) in Sweden..$^{9,10}$ The contrast of a MIEZE signal is sensitive to differences in the length of the neutron flight path. This drawback is very severe and it has hampered the use of MIEZE spectrometers in steady-state neutron sources. Several test experiments and simulations of MIEZE spectrometers have been conducted. ${ }^{11-15}$ The geometrical problem between the sample and detector has been discussed; however, the advantages of the MIEZE technique combined with the TOF method (TOF-MIEZE) have not been explicitly pointed out yet. By using a short pulsed beam, the effect of mismatching from the optimal position of the components does not change the contrast of a TOF-MIEZE signal, but rather, the effective frequency. In the present study, we clarify these characteristics which are essentially different from MIEZE signals by a continuous beam, using a basic formula for the TOF-MIEZE technique and a simple experimental demonstration.

\section{FORMALIZATION OF TOF-MIEZE}

Figure 1 shows an energy diagram of a simple setup of a MIEZE spectrometer using two resonance spin flippers (RSFs) with different radio frequencies of $\omega_{1}$ and $\omega_{2}\left(\omega_{1}<\omega_{2}\right)$. The setup consists of a polarizer, two RSFs, an analyzer, and a detector. Both RSFs are operated as $\pi / 2$ flippers, which change a neutron's spin eigen state with a probability of $1 / 2$ and make a superposition of up- and down-spin states with an energy difference corresponding to the frequency of the RSF. The wave function of a neutron after the polarizer is expressed as follows: 


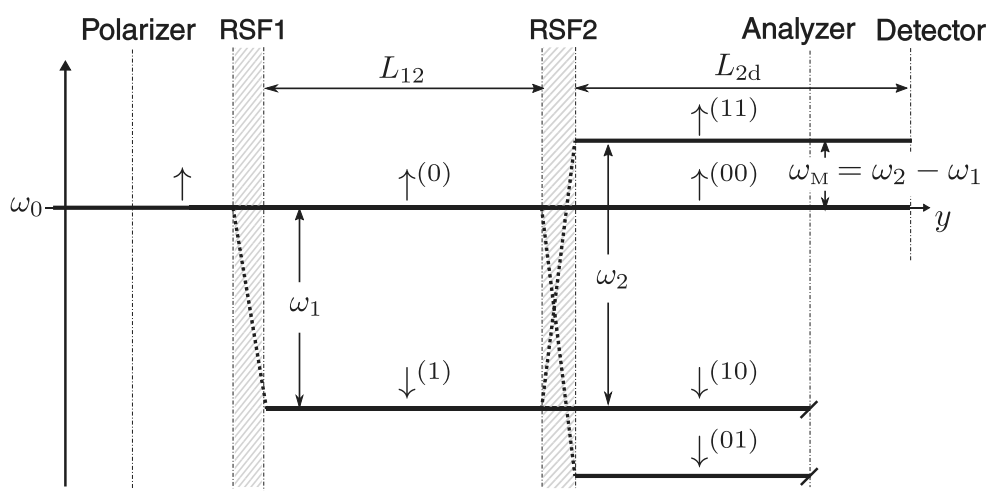

FIG. 1. Neutron energy changes in units of $\hbar$ along with the neutron flight path in a simple MIEZE setup. Symbols of $\uparrow$ and $\downarrow$ indicate up- and down-spin eigenstates, respectively, and their superscripts are indices of flipped (1) or non-flipped (0) components at RSF1 or RSF2.

$$
|\uparrow\rangle=\left(\begin{array}{l}
1 \\
0
\end{array}\right) \mathrm{e}^{\mathrm{i}\left(k_{0} y-\omega_{0} t\right)},
$$

where $\omega_{0}$ is the kinetic energy of the incident neutron in units of $\hbar, k_{0}$ is the wavenumber of the incident neutron $k_{0}=\sqrt{2 m_{\mathrm{n}} \omega_{0} / \hbar}, m_{\mathrm{n}}$ is the neutron mass, and $\hbar$ is the reduced Planck constant. A $\pi / 2$ flipper creates a superposition of $\left|\downarrow^{(1)}\right\rangle$ and $\left|\uparrow^{(0)}\right\rangle$. The superscripts (1) or (0) indicate whether or not a neutron is flipped, respectively, by the first resonance spin flipper RSF1. After applying RSF1, the wave function becomes $^{16,17}$

$$
\left|\uparrow^{(0)}\right\rangle+\left|\downarrow^{(1)}\right\rangle=\frac{1}{\sqrt{2}}\left(\begin{array}{c}
1 \\
\mathrm{e}^{\mathrm{i}\left(-\omega_{1} y / v+\omega_{1} t-\pi / 2\right)}
\end{array}\right) \mathrm{e}^{\mathrm{i}\left(k_{0} y-\omega_{0} t\right)},
$$

where $v$ is the neutron velocity $v=\sqrt{2 \hbar \omega_{0} / m_{\mathrm{n}}}$, and we used the following approximation: $\sqrt{2 m_{\mathrm{n}}\left(\omega_{0}-\omega_{1}\right) / \hbar} \simeq k_{0}-\omega_{1} / v$ because $\omega_{1} \ll \omega_{0}$. This approximation is valid for a wide energy range of neutrons such as thermal and cold neutrons, in almost every realistic experimental condition. Even for very cold neutrons and high frequencies in $\mathrm{MHz}$ order, the fraction $\omega_{1} / \omega_{0}$ is less than $10^{-3}$. The spin flipped component $\left|\downarrow^{(1)}\right\rangle$ loses a total energy of $\hbar \omega_{1}$. After the second $\pi / 2$ flipper RSF2 with a frequency of $\omega_{2}$, we can consider four components: $\left|\uparrow^{(11)}\right\rangle$, $\left|\uparrow^{(00)}\right\rangle,\left|\downarrow^{(10)}\right\rangle$, and $\left|\downarrow^{(01)}\right\rangle$ as shown in Fig. 1. The wave function becomes

$$
\begin{aligned}
\left|\uparrow^{(00)}\right\rangle+\left|\uparrow^{(11)}\right\rangle+\left|\downarrow^{(10)}\right\rangle+\left|\downarrow^{(01)}\right\rangle \\
=\frac{1}{2}\left(\begin{array}{c}
1+\mathrm{e}^{\mathrm{i} \phi_{12}} \mathrm{e}^{\mathrm{i}\left(\omega_{2}-\omega_{1}\right) y / v} \mathrm{e}^{-\mathrm{i}\left[\left(\omega_{2}-\omega_{1}\right) t+\pi / 2\right]} \\
\mathrm{e}^{\mathrm{i} \phi_{12}} \mathrm{e}^{-\mathrm{i} \omega_{1} y / v} \mathrm{e}^{\mathrm{i} \omega_{1} t}+\mathrm{e}^{-\mathrm{i} \omega_{2} y / v} \mathrm{e}^{\mathrm{i}\left(\omega_{2} t-\pi / 2\right)}
\end{array}\right) \\
\quad \times \mathrm{e}^{\mathrm{i}\left(k_{0} y-\omega_{0} t\right)},
\end{aligned}
$$

where $\phi_{12}$ is the phase difference depending on the difference of the kinetic energy and the flight path length between RSF1 and RSF2, $L_{12}$, given by $\phi_{12}=-\omega_{1} L_{12} / v$. After the spin analyzer, we obtain one spin state with two different energies: $\hbar \omega_{0}$ and $\hbar\left(\omega_{0}+\omega_{2}-\omega_{1}\right)$. The phase difference at the detector is expressed as follows:

$$
\phi\left(t_{\mathrm{d}}\right)=-\left(\omega_{2}-\omega_{1}\right) t_{\mathrm{d}}-\frac{\omega_{1}}{v} L_{12}+\frac{\left(\omega_{2}-\omega_{1}\right)}{v} L_{2 \mathrm{~d}},
$$

where $L_{2 \mathrm{~d}}$ is the flight path length between RSF2 and the detector. The time point $t_{\mathrm{d}}$ is the time when a neutron is de- tected. The detected neutron intensity $I$ is calculated to be $I \propto$ ||$\left.\left.\uparrow^{(00)}\right\rangle+\left.\left|\uparrow^{(11)}\right\rangle\right|^{2}=\left|\left(1+\mathrm{e}^{\mathrm{i} \phi\left(t_{\mathrm{d}}\right)}\right) / \sqrt{2}\right| \uparrow\right\rangle\left.\right|^{2}=\left(1+\cos \left[\phi\left(t_{\mathrm{d}}\right)\right]\right) / 2$.

Let us consider a no sample case, where the velocities and directions of neutrons do not change. Equation (4) can be written as

$$
\phi_{\mathrm{el}}\left(t_{\mathrm{d}}\right)=-\omega_{\mathrm{M}} t_{\mathrm{d}}+\Delta \omega \frac{L_{0 \mathrm{~d}}}{v} .
$$

Here, we use $\omega_{M}=\omega_{2}-\omega_{1}$ for simplicity and define a "detuning frequency" as

$$
\Delta \omega=\frac{-\omega_{1} L_{12}+\left(\omega_{2}-\omega_{1}\right) L_{2 \mathrm{~d}}}{L_{0 \mathrm{~d}}},
$$

where $L_{0 \mathrm{~d}}$ is the total flight path length from the origin of the pulsed beam to the detector. The phase that depends on neutron velocities will be canceled when $\Delta \omega=0$, which is called the MIEZE condition. In experiments using a pulsed beam, the neutron velocity $v$ can be obtained as

$$
v=\frac{L_{0 \mathrm{~d}}}{t_{0 \mathrm{~d}}},
$$

by measuring the total flight path length $L_{0 \mathrm{~d}}$ and the time-offlight $t_{0 \mathrm{~d}}=t_{\mathrm{d}}-t_{0}\left(t_{0}\right.$ is the time origin of the pulse beam). Using this expression for $v$, we obtain

$$
\phi_{\mathrm{el}}\left(t_{\mathrm{d}}\right)=-\left(\omega_{\mathrm{M}}-\Delta \omega\right) t_{\mathrm{d}}-\Delta \omega t_{0} .
$$

Here, we assume the time origins $t_{0}$ for each neutron are precisely fixed and the term $-\Delta \omega t_{0}$ causes a constant shift in the phase difference; therefore, the contrast of the TOFMIEZE signal does not decrease even at an off-MIEZE condition $(\Delta \omega \neq 0)$ and the neutron intensity modulates as a perfect sinusoidal function of $t_{\mathrm{d}}$ with a frequency of $\omega_{M}-\Delta \omega$. The detected intensity as a function of $t_{\mathrm{d}}$ is given by

$$
I\left(t_{\mathrm{d}}\right)=\int \mathrm{d} v \frac{I_{0}(v)}{2}\left(1+\cos \left[\left(\omega_{\mathrm{M}}-\Delta \omega\right) t_{\mathrm{d}}\right]\right),
$$

where $I_{0}(v)$ is the spectrum of the direct beam without RSFs.

Next, we consider the TOF-MIEZE signal with an inelastic scattering sample. The velocity change at a sample is given as $\delta v=v_{\mathrm{f}}-v_{\mathrm{i}}$, where $v_{\mathrm{i}}$ and $v_{\mathrm{f}}$ are the neutron velocities before and after the sample, respectively. The time-of-flight, $t_{0 \mathrm{~d}}$ is expressed as the following, keeping only the first order 
of $\delta v / v_{\mathrm{i}} \ll 1$,

$$
t_{0 \mathrm{~d}}=\frac{L_{01}+L_{12}+L_{2 \mathrm{~s}}}{v_{\mathrm{i}}}+\frac{L_{\mathrm{sd}}}{v_{\mathrm{f}}} \simeq \frac{L_{0 \mathrm{~d}}}{v_{\mathrm{i}}}-\frac{L_{\mathrm{sd}}}{v_{\mathrm{i}}^{2}} \delta v,
$$

where $L_{0 \mathrm{~d}}=L_{01}+L_{12}+L_{2 \mathrm{~s}}+L_{\mathrm{sd}}$, and $L_{\mathrm{sd}}$ is the flight path length between the sample position and the detector. In most NSE measurements, the relative value of $\delta v / v_{\mathrm{i}}$ is in the order of $10^{-5}$ to $10^{-3}$. Eq. (10) can be regarded as a quadratic equation in $v_{\mathrm{i}}$ and its solutions are

$$
v_{\mathrm{i}}=\frac{L_{0 \mathrm{~d}}}{2 t_{0 \mathrm{~d}}}\left[1 \pm \sqrt{1-\frac{4 t_{0 \mathrm{~d}} L_{\mathrm{sd}} \delta v}{L_{0 \mathrm{~d}}^{2}}}\right] .
$$

The solution with a plus sign has physical significance and $4 t_{0 \mathrm{~d}} L_{\mathrm{sd}} \delta v / L_{0 \mathrm{~d}}^{2} \ll 1$. Thus, $v_{\mathrm{i}}$ is expressed as

$$
v_{\mathrm{i}} \simeq \frac{L_{0 \mathrm{~d}}}{t_{0 \mathrm{~d}}}+\frac{L_{\mathrm{sd}}}{L_{0 \mathrm{~d}}} \delta v
$$

The phase difference with an inelastic scattering is approximated in the same manner in Eq. (10),

$$
\phi_{\text {inel }}\left(t_{\mathrm{d}}\right) \simeq-\omega_{\mathrm{M}} t_{\mathrm{d}}+\Delta \omega \frac{L_{0 \mathrm{~d}}}{v_{\mathrm{i}}}+\frac{\omega_{\mathrm{M}} L_{\mathrm{sd}}}{v_{\mathrm{i}}^{2}} \delta v .
$$

By substituting Eq. (12) into Eq. (13), we obtain

$\phi_{\text {inel }}\left(t_{\mathrm{d}}\right) \simeq-\left(\omega_{\mathrm{M}}-\Delta \omega\right) t_{\mathrm{d}}-\Delta \omega t_{0}+\left(\omega_{\mathrm{M}}-\Delta \omega\right) \frac{L_{\mathrm{sd}}}{v_{0}^{2}} \delta v$,

where we use $L_{0 \mathrm{~d}} / t_{0 \mathrm{~d}}=v_{0}$ for simplicity and omit the higher order terms of $L_{\mathrm{sd}} \delta v /\left(L_{0 \mathrm{~d}} v_{0}\right) \ll 1$ because $L_{\mathrm{sd}}<L_{0 \mathrm{~d}}$ and $\delta v / v_{0}$ $\simeq \delta v / v_{\mathrm{i}} \ll 1$.

Let us represent a change in the phase difference between the elastic and inelastic cases with the following style:

$$
\phi_{\text {inel }}-\phi_{\mathrm{el}}=\omega \tau_{\mathrm{M}},
$$

where $\omega$ denotes an energy exchange by the sample defined as $\hbar \omega=m_{\mathrm{n}}\left(v_{\mathrm{f}}^{2}-v_{\mathrm{i}}^{2}\right) / 2 \simeq m_{\mathrm{n}} v_{\mathrm{i}} \delta v \simeq m_{\mathrm{n}} v_{0} \delta v$. Thus, an index of the energy resolution of NSE instruments, so-called Fourier time, is introduced as

$$
\tau_{\mathrm{M}}=\frac{\phi_{\text {inel }}-\phi_{\mathrm{el}}}{\omega}=\frac{\hbar L_{\mathrm{sd}}}{m_{\mathrm{n}} v_{0}^{3}}\left(\omega_{\mathrm{M}}-\Delta \omega\right) .
$$

Although the effective frequency is shifted by $\Delta \omega$ in an offMIEZE condition, the Fourier time of the TOF-MIEZE spectrometer is proportional to the effective frequency $\omega_{M}-\Delta \omega$, the sample-detector distance $L_{\mathrm{sd}}$, and the third power of the neutron wavelength, similar to other NSE methods.

\section{EXPERIMENT}

We have performed experiments to demonstrate the characteristics of TOF-MIEZE signals with different time widths of pulsed neutron beams. Figure 2 shows a schematic of the TOF-MIEZE instrument installed at the beam port of verycold neutrons (VCNs) at the High Flux Reactor of the Institut Laue-Langevin (ILL). We selected a VCN beam with a wavelength of $\lambda \simeq 3.5$ to $9 \mathrm{~nm}$ by a disk chopper. The total flight path length $L_{0 \mathrm{~d}}$ was $3.0 \mathrm{~m}$. The distance between RSF1 and RSF2 $L_{12}$ was $1.5 \mathrm{~m}$. The distance from RSF2 to the detector $L_{2 \mathrm{~d}}$ was $1.0 \mathrm{~m}$. The chopper disk has an adjustable open window to change the pulse width $\Delta t_{0}$. The time width is estimated as $\Delta t_{0}=D /(2 \pi R f)$, where $D$ is the open window width, $R$ is the radius of the disk, and $f$ is the frequency of rotation. The frequency $f$ was set at $12.5 \mathrm{~Hz}$ and $R=163 \mathrm{~mm}$. $\Delta t_{0}$ was estimated as $0.7 \mathrm{~ms}$ and $2.7 \mathrm{~ms}$ for $D=9 \mathrm{~mm}$ and $35 \mathrm{~mm}$, respectively.

We used $m=4$ polarizing $\mathrm{Fe} / \mathrm{SiGe}$ super-mirrors coated on silicon wafers by an ion beam sputtering machine ${ }^{18}$ as the polarizer and analyzer. The polarizing efficiency was higher than $90 \%$ for neutrons with a wavelength of $5.0 \mathrm{~nm}$. We used a two-dimensional time-sensitive detector consisting of a 5 in. position-sensitive photo multiplier ${ }^{19}$ and a ${ }^{6} \mathrm{Li}$ enriched glass scintillator with a thickness of $0.2 \mathrm{~mm}$.

We constructed the experimental setup of TOF-MIEZE by a pair of RSFs, which consist of a static field and an oscillating field with radio frequency (RF). When the RF and static field satisfies the magnetic resonance condition, the probability of spin flip is given by $P=\sin ^{2}\left(\left|\mu_{\mathrm{n}}\right| B_{\mathrm{r}} \ell /(\hbar v)\right)$, where $\mu_{\mathrm{n}}$ is the neutron magnetic moment, $B_{\mathrm{r}}$ is the amplitude of oscillating field, $\ell$ is the length of RF region, and $v$ is the neutron velocity. The cases of $P=1$ and $1 / 2$ are called the $\pi$ and $\pi / 2$ flip condition, respectively. By modulating $B_{\mathrm{r}}$ to be inversely proportional to neutron's spent time in the oscillating field, the RSF is applicable to a pulsed white beam. ${ }^{20,21}$ We determined the flipping efficiency in the different open widths of $D$ $=9 \mathrm{~mm}$ and $35 \mathrm{~mm}$, and confirmed that the efficiency change was negligible. The frequencies of RSF1 and RSF2 were set as $\omega_{1} / 2 \pi=10 \mathrm{kHz}$ and $\omega_{2} / 2 \pi=11 \mathrm{kHz}$, respectively. An effective frequency of $1 \mathrm{kHz}$ is lower than usual MIEZE frequencies by two orders of magnitude. In this setup, the Fourier time was up to a few nanoseconds, which was poor as for a highresolution spectrometer. As this experiment was not aiming for high-resolution, the $1 \mathrm{kHz}$ MIEZE oscillation was enough for this proof-of-principle experiment.

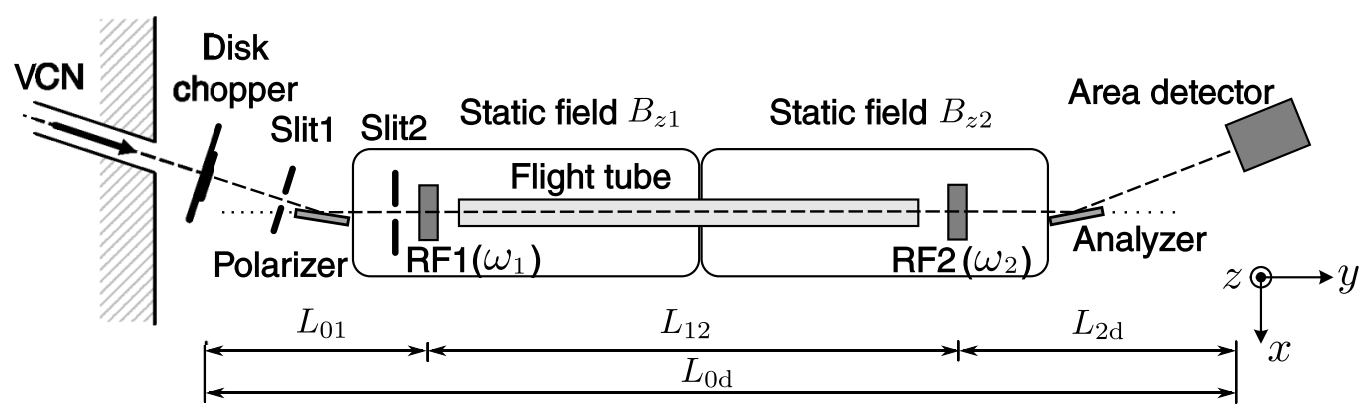

FIG. 2. Top view of the setup of the TOF-MIEZE experiment at the PF2 VCN port of the ILL reactor. 


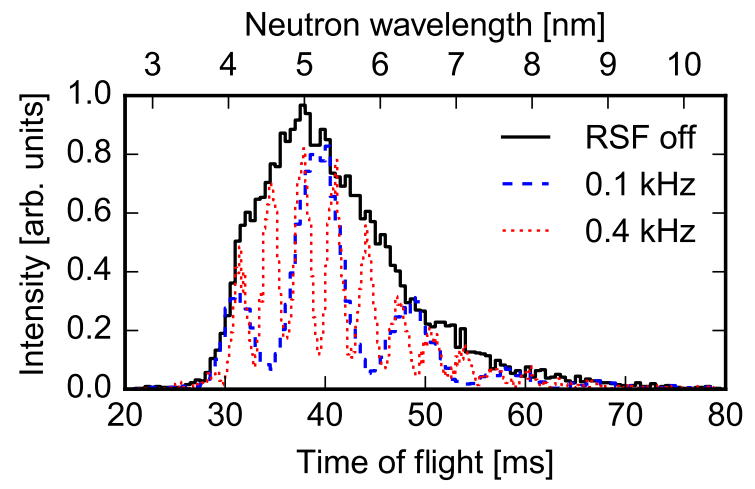

FIG. 3. TOF spectrum of the direct beam (solid line). TOF-MIEZE signals with effective frequency of $0.1 \mathrm{kHz}$ (dashed line) and $0.4 \mathrm{kHz}$ (dotted line) in the MIEZE condition.

As shown in Fig. 2, we applied two static fields over the flight path. The magnitudes of static fields $B_{z 1}$ and $B_{z 2}$ almost corresponded to the magnetic resonance conditions for $\omega_{1}$ and $\omega_{2}$, respectively. With these fields, the phase difference between up- and down-spin states was nearly zero, and hence, $\Delta \omega$ was nearly zero independent of the relation between $L_{12}$ and $L_{2 \mathrm{~d}}$.

\section{RESULTS}

Figure 3 shows TOF-MIEZE signals with effective frequencies of 0.1 and $0.4 \mathrm{kHz}$ and a spectrum of the polarized VCN beam without RSF operation. The frequency of RSF1 was

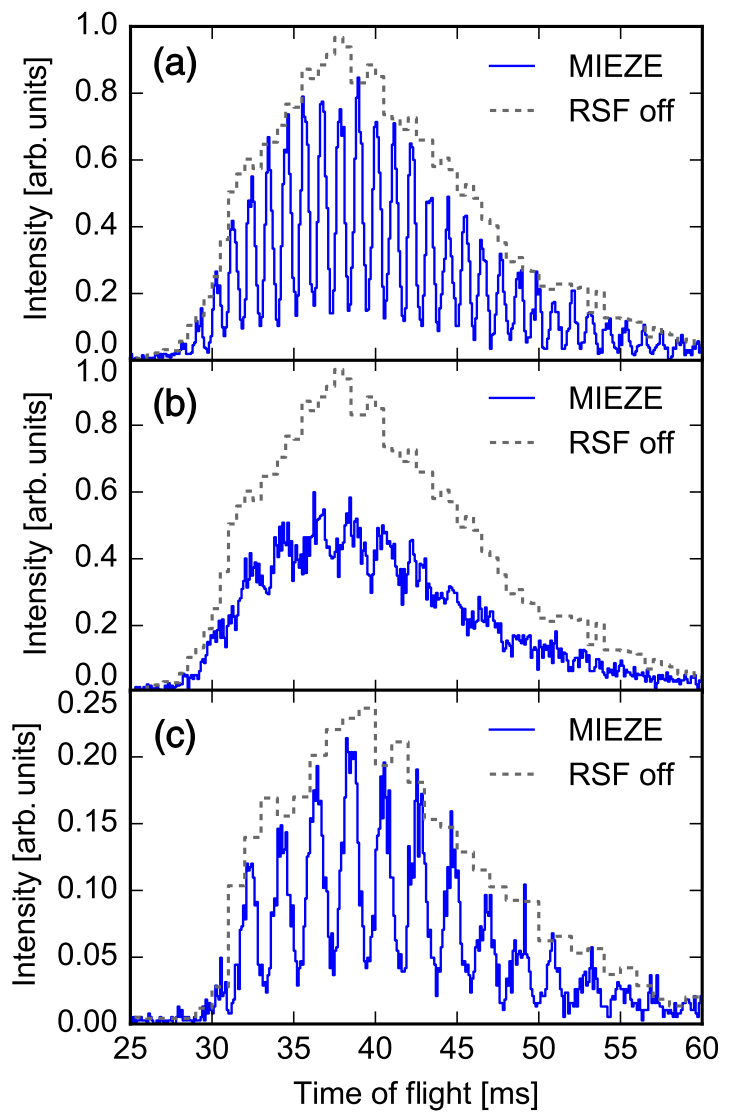

FIG. 4. TOF-MIEZE signal with a frequency of $1 \mathrm{kHz}$; (a) on-MIEZE condition, (b) off-MIEZE condition with the wide pulse width $\Delta t_{0}=2.7 \mathrm{~ms}$, and (c) off-MIEZE condition signal with the narrow pulse width $\Delta t_{0}=0.7 \mathrm{~ms}$.
TABLE I. Effective frequency and contrast of TOF-MIEZE signal with the pulse width and the settings for MIEZE condition.

\begin{tabular}{lccc}
\hline \hline MIEZE condition & $\begin{array}{c}\text { Pulse width } \\
(\mathrm{ms})\end{array}$ & $\begin{array}{c}\text { Frequency } \\
(\mathrm{kHz})\end{array}$ & $\begin{array}{c}\text { Contrast } \\
\text { (averaged) }\end{array}$ \\
\hline (a) on, $\Delta \omega / 2 \pi=0 \mathrm{kHz}$ & 2.7 & 0.92 & 0.68 \\
(b) off, $\Delta \omega / 2 \pi=0.5 \mathrm{kHz}$ & 2.7 & $\mathrm{~N} / \mathrm{A}$ & $\mathrm{N} / \mathrm{A}$ \\
(c) off, $\Delta \omega / 2 \pi=0.5 \mathrm{kHz}$ & 0.7 & 0.48 & 0.63 \\
\hline \hline
\end{tabular}

set at $\omega_{1} / 2 \pi=10 \mathrm{kHz}$, while the frequencies of RSF2 were set to $\omega_{2} / 2 \pi=10.1 \mathrm{kHz}$ and $\omega_{2} / 2 \pi=10.4 \mathrm{kHz}$. TOF-MIEZE signals with the frequency of $\omega_{\mathrm{M}}=\omega_{2}-\omega_{1}$ were observed.

Figure 4(a) shows a TOF-MIEZE signal with an effective frequency of $1 \mathrm{kHz}$ on the MIEZE condition. In the setup of Fig. 4(b), an off-MIEZE condition was realized by changing the magnitude of the static field in a part of the flight path between RSF1 and RSF2, and the detuning frequency was evaluated as approximately $0.5 \mathrm{kHz}$ from Eq. (6). The TOFMIEZE signal of Fig. 4(b) was deteriorated. The setup of Fig. 4(c) was at the same off-MIEZE condition as (b) but we changed the pulse width to $\Delta t_{0}=0.7 \mathrm{~ms}$ from $\Delta t_{0}=2.7 \mathrm{~ms}$ of (a) and (b). The signal contrast of the off-MIEZE condition (c) was comparable to that of the on-MIEZE condition (a), and the effective frequency was clearly shifted from $1 \mathrm{kHz}$ to $0.48 \mathrm{kHz}$. The MIEZE conditions, pulse widths, and observed parameters are summarized in Table I.

\section{DISCUSSION}

The dephasing effects of TOF-MIEZE signals can be understood by the deviations of the detection time and the pulse width. In this study, we focus on the effect of pulse width $\Delta t_{0}$ to clarify the effect of the term $-\Delta \omega t_{0}$ in Eq. (8). The phase deviation due to the pulse width is given by

$$
\left|\frac{\partial \phi}{\partial t_{0}}\right| \Delta t_{0}=\Delta \omega \Delta t_{0}
$$

and the pulse width should satisfy the following constraint to maintain the contrast:

$$
\Delta t_{0} \ll \frac{2 \pi}{\Delta \omega} .
$$

For the pulse width of $\Delta t_{0}=0.7 \mathrm{~ms}$ and $2.7 \mathrm{~ms}$, the allowable detuning frequencies were estimated as $\Delta \omega / 2 \pi=1.4 \mathrm{kHz}$ and $0.37 \mathrm{kHz}$, respectively, from Eq. (18). Equation (18) explained the difference in the contrast of the TOF-MIEZE signals shown in Figs. 4(b) and 4(c). This implies that narrower pulse widths promise more robust TOF-MIEZE signals even in an offMIEZE condition. For a sufficiently short pulse width, the detuning effect is manifested in a frequency shift, not in contrast decreasing.

From Eq. (8), deviation of the detection time causes a dephasing

$$
\left|\frac{\partial \phi}{\partial t_{\mathrm{d}}}\right| \Delta t_{\mathrm{d}}=\left(\omega_{\mathrm{M}}-\Delta \omega\right) \Delta t_{\mathrm{d}} .
$$

Therefore the requirement for maintaining signal contrast is

$$
\Delta t_{\mathrm{d}} \ll \frac{2 \pi}{\omega_{\mathrm{M}}-\Delta \omega} \simeq \frac{2 \pi}{\omega_{\mathrm{M}}} .
$$


The deviation of the detection time is caused by the deviation of neutron flight path length and velocity change by a sample. Considerable care should be taken for the deviation of $L_{\mathrm{sd}}$ by a sample volume in the MIEZE technique., ${ }^{9,10,22}$ Using the formula, we can separate the problem of matching of the MIEZE condition from the geometrical problem of the deviation of flight path lengths, and evaluate the resolution function of a TOF-MIEZE spectrometer precisely.

\section{CONCLUSION}

The contrast of a TOF-MIEZE signal is robust against the misalignment of optical components when the pulse width is small enough. Although the effective frequency is shifted in an off-MIEZE condition, the intermediate scattering function $S(Q, \tau)$ can be obtained similarly for the MIEZE condition. The shift of the effective frequency is useful to optimize the experimental setup for the MIEZE condition. The robustness of signal contrast is a great advantage of TOF-MIEZE spectroscopy with pulsed neutron beam. The characteristics of the TOF-MIEZE technique can be beneficial for various experiments using resonance spin flippers in neutron scattering spectroscopy and in high-precision fundamental neutron physics. ${ }^{23,24}$

\section{ACKNOWLEDGMENTS}

Preliminary tests of TOF-MIEZE experiments were conducted at BL10 and BL05 of J-PARC/MLF. The authors would like to thank T. Brenner at PF2 ILL for his technical support. This work was supported by JSPS KAKENHI Grant Nos. JP23360428 and JP15K13405, and the Photon and Quantum Basic Research Coordinated Development Program from the Ministry of Education, Culture, Sports, Science and Technology, Japan. Certain device developments were supported by the program of Development of System and Technology for Advanced Measurement Analysis (SENTAN), JST and the Neutron Scattering Program Advisory Committee of IMSS, KEK (Proposal Nos. 2009S07 and 2014S07). One of the authors (T.O.) was financially supported by Grant-in-Aid for JSPS fellows.
${ }^{1}$ F. Mezei, Z. Phys. A: Hadrons Nucl. 255, 146 (1972).

${ }^{2}$ Neutron Spin Echo, Lecture Notes in Physics Vol. 128, edited by F. Mezei (Springer-Verlag, Berlin, 1980).

${ }^{3}$ R. Golub and R. Gähler, Phys. Lett. A 123, 43 (1987).

${ }^{4}$ R. Gähler and R. Golub, Z. Phys. B 65, 269 (1987).

${ }^{5}$ R. Gähler, R. Golub, and T. Keller, Physica B 180, 899 (1992).

${ }^{6}$ R. Georgii, G. Brandl, N. Arend, W. Häußler, A. Tischendorf, C. Pfleiderer, P. Böni, and J. Lal, Appl. Phys. Lett. 98, 073505 (2011).

${ }^{7}$ Y. Kawabata, M. Hino, M. Kitaguchi, H. Hayashida, S. Tasaki, T. Ebisawa, D. Yamazaki, R. Maruyama, H. Seto, M. Nagao, and T. Kanaya, Physica B 385-86, 1122 (2006).

${ }^{8}$ M. Hino, T. Oda, M. Kitaguchi, N. L. Yamada, H. Sagehashi, Y. Kawabata, and H. Seto, Phys. Procedia 42, 136 (2013).

${ }^{9}$ G. Brandl, R. Georgii, W. Häußler, S. Muehlbauer, and P. Böni, Nucl. Instrum. Methods Phys. Res., Sect. A 654, 394 (2011).

${ }^{10}$ T. Weber, G. Brandl, R. Georgii, W. Häußler, S. Weichselbaumer, and P. Böni, Nucl. Instrum. Methods Phys. Res., Sect. A 713, 71 (2013).

${ }^{11}$ W. Besenböck, R. Gähler, P. Hank, R. Kahn, M. Köppe, C. H. de Novion, W. Petry, and J. Wuttke, J. Neutron Res. 7, 65 (1998).

${ }^{12}$ H. Hayashida, M. Kitaguchi, M. Hino, Y. Kawabata, and T. Ebisawa, Physica B 397, 144 (2007)

${ }^{13}$ W. Häußler, P. Böni, M. Klein, C. J. Schmidt, U. Schmidt, F. Groitl, and J. Kindervater, Rev. Sci. Instrum. 82, 045101 (2011).

${ }^{14}$ M. Bleuel, M. Broll, E. Lang, K. Littrell, R. Gähler, and J. Lal, Physica B 371, 297 (2006).

${ }^{15}$ G. Brandl, J. Lal, J. Carpenter, L. Crow, L. Robertson, R. Georgii, P. Böni, and M. Bleuel, Nucl. Instrum. Methods Phys. Res., Sect. A 667, 1 (2012).

${ }^{16}$ R. Golub, R. Gähler, and T. Keller, Am. J. Phys. 62, 779 (1994).

${ }^{17}$ D. Yamazaki, Nucl. Instrum. Methods Phys. Res., Sect. A 488, 623 (2002).

${ }^{18}$ M. Hino, T. Oda, M. Kitaguchi, N. L. Yamada, S. Tasaki, and Y. Kawabata, Nucl. Instrum. Methods Phys. Res., Sect. A 797, 265 (2015).

${ }^{19}$ K. Hirota, T. Shinohara, K. Ikeda, K. Mishima, T. Adachi, T. Morishima, S. Satoh, T. Oku, S. Yamada, H. Sasao, J. Suzuki, and H. M. Shimizu, Phys. Chem. Chem. Phys. 7, 1836 (2005).

${ }^{20}$ R. Maruyama, T. Ebisawa, S. Tasaki, M. Hino, M. Takeda, T. Kawai, Y. Kawabata, and K. Sakai, Physica B 335, 238 (2003).

${ }^{21}$ H. Hayashida, M. Kitaguchi, M. Hino, Y. Kawabata, R. Maruyama, and T. Ebisawa, Nucl. Instrum. Methods Phys. Res., Sect. A 574, 292 (2007).

${ }^{22}$ H. Hayashida, M. Hino, M. Kitaguchi, Y. Kawabata, and N. Achiwa, Meas. Sci. Technol. 19, 034006 (2008).

${ }^{23}$ P. N. Seo, L. Barrón-Palos, J. D. Bowman, T. E. Chupp, C. Crawford, M. Dabaghyan, M. Dawkins, S. J. Freedman, T. Gentile, M. T. Gericke, R. C. Gillis, G. L. Greene, F. W. Hersman, G. L. Jones, M. Kandes, S. Lamoreaux, B. Lauss, M. B. Leuschner, R. Mahurin, M. Mason, J. Mei, G. S. Mitchell, H. Nann, S. A. Page, S. I. Penttilä, W. D. Ramsay, A. Salas Bacci, S. Santra, M. Sharma, T. B. Smith, W. M. Snow, W. S. Wilburn, and H. Zhu, Phys. Rev. Spec. Top.-Accel. Beams 11, 084701 (2008).

${ }^{24}$ H. Abele, T. Jenke, H. Leeb, and J. Schmiedmayer, Phys. Rev. D 81, 065019 (2010). 